\title{
Bouveret syndrome and pancreatic acinar cell carcinoma
}

Bouveret syndrome and pancreatic acinar cell carcinoma are two rare conditions of the gastrointestinal tract [1-4]. Herein, we present a case in which both conditions occurred in the same patient.

A 77-year-old man with diabetes and a history of cholelithiasis with recurrent episodes of biliary pain was referred for evaluation of epigastric pain, anorexia, nausea, bloating, and weight loss during the previous 6 weeks. Results of laboratory tests were normal. Magnetic resonance imaging demonstrated a solid lesion in the pancreatic head, and the patient was sent for evaluation with endoscopic ultrasound-guided fine-needle aspiration (EUS-FNA).

Unexpectedly, at the beginning of the procedure, a huge hard gallstone was found to be completely obstructing the pylorus, and was impacted in the first portion of the duodenum. The stone was pulled back into the stomach using a polypectomy snare, and was broken into small fragments with a metal lithotriptor. The small fragments were still too large to be removed via the mouth, as they impacted below the cardia, and were therefore left in the stomach. The patient was asymptomatic, and a small-bowel contrast radiogram some days later confirmed the elimination of the stones.

After the removal of the pyloric gallstone, EUS-FNA was performed through de duodenum. A nodule with regular margins and some small cystic areas, measuring $2.4 \times 1.8 \mathrm{~cm}$, was found in the head of the pancreas. FNA was performed using a 22-gauge needle, with a total of four passes, and the aspirated material was prepared as cell blocks.

Histopathological evaluation and immunohistochemical panel for acinar-specific markers confirmed the diagnosis of an acinar cell carcinoma of the pancreas. Immunohistochemistry was positive for alpha-1-antichymotrypsin, alpha-1-trypsin, and CKM (AE1/AE3/PCK26). In addition, Ki-67 revealed a proliferation index of $70 \%$.
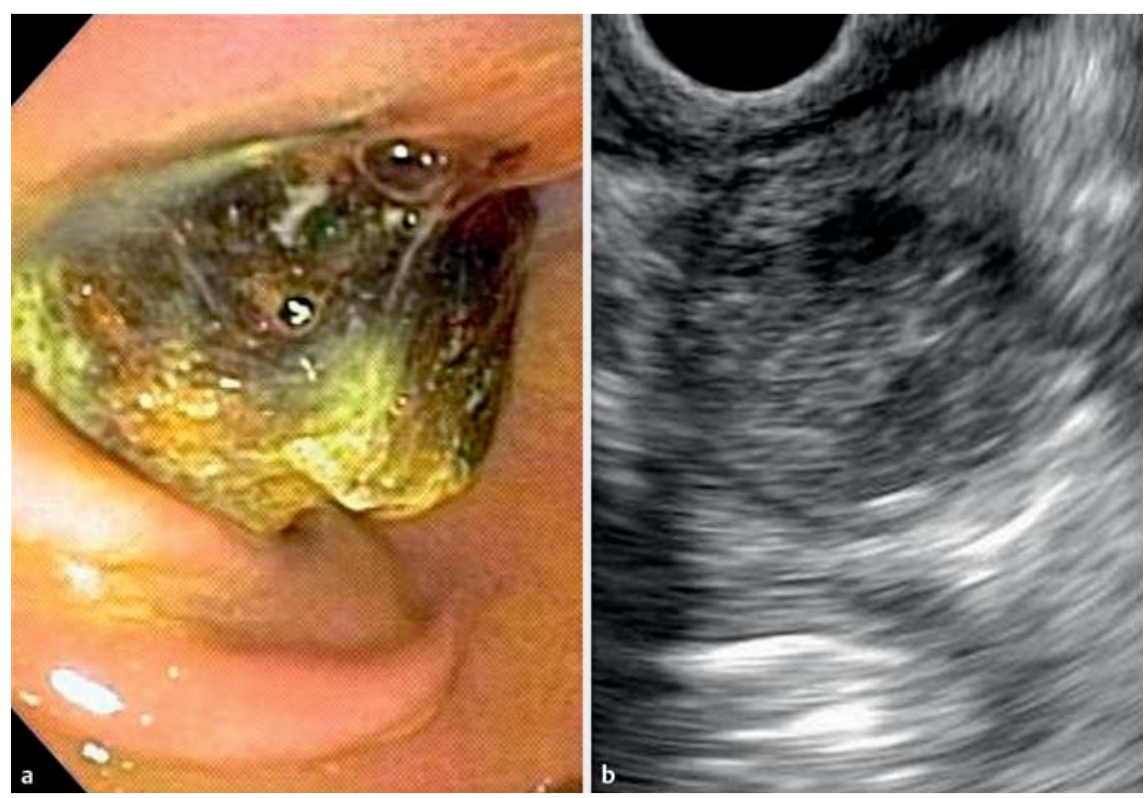

- Fig. 1 A patient with Bouveret syndrome and pancreatic acinar cell carcinoma. a A gallstone completely obstructed the pylorus in a patient with cholelithiasis and recurrent episodes of biliary pain. $\mathbf{b}$ Linear-array endosonography demonstrated a pancreatic nodule in the head of the pancreas, with well-defined borders and some cystic areas, measuring $2.4 \times 1.8 \mathrm{~cm}$. Fine-needle aspiration confirmed the diagnosis of an acinar cell carcinoma.
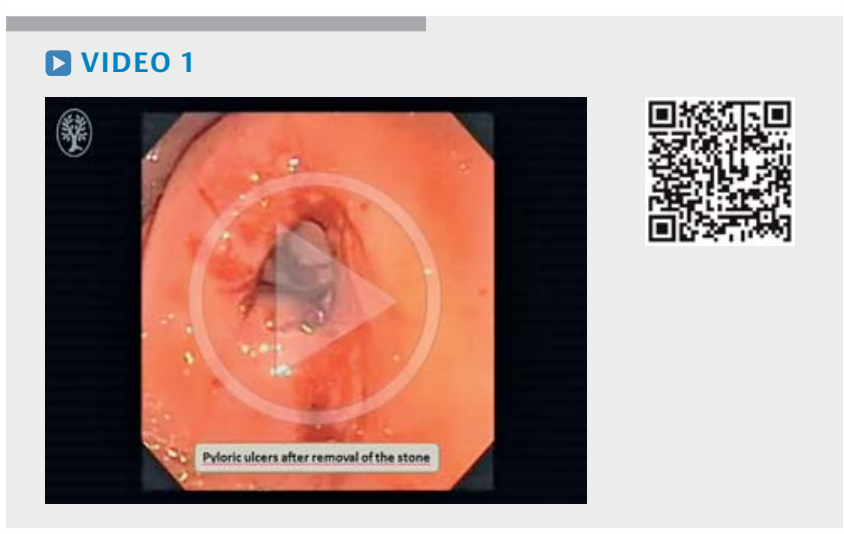

Video 1: Bouveret syndrome constitutes less than $3 \%$ of all gallstone-induced ileus due to the migration of a gallstone through a cholecystoduodenal fistula and its impaction in the pylorus or duodenum. Endoscopic management is the first therapeutic option. In this case, the gallstone was removed from the pylorus to the stomach using a polypectomy snare and broken into small pieces with a metal lithotriptor.

Pancreatic acinar cell carcinoma represents less than $1 \%$ of pancreatic tumors in adults. The diagnosis is based on morphology and immunohistochemical detection of acinar-specific markers. In this case, the panel was positive for alpha-1-antichymotrypsin, alpha-1-trypsin, and CKM (AE1/AE3/PCK26). Surgery is the treatment of choice for this tumor. 
The patient was initially treated by chemotherapy with 5-fluorouracil. There was no clinical response, and he underwent a Whipple procedure some months later. A R0 resection was performed, and the patient's clinical course has been uneventful to date.

Endoscopy_UCTN_Code_CCL_1AB_2AZ_3AZ

Endoscopy_UCTN_Code_CCL_1AF_2AZ_3AB

\section{Competing interests}

None
The Authors

César Vivian Lopes ${ }^{1}$, Fernando Krebs Cirne Lima $^{2}$, Antônio Atalíbio Hartmann ${ }^{3}$

1 Department of Gastroenterology and Digestive Endoscopy, Santa Casa Hospital, Porto Alegre, Brazil

2 Department of General Surgery, Santa Casa Hospital, Porto Alegre, Brazil

3 Department of Pathology, Santa Casa Hospital, Porto Alegre, Brazil

\section{Corresponding author}

\section{César Vivian Lopes, MD, PhD}

Department of Gastroenterology and

Digestive Endoscopy, Santa Casa Hospital, Rua Prof. Cristiano Fischer 668/1001,

C.E.P. 91.410-000 Porto Alegre-RS, Brazil Fax: +55-51-33388054

drcvlopes@gmail.com
References

[1] Cappell MS, Davis M. Characterization of Bouveret's syndrome: a comprehensive review of 128 cases. Am J Gastroenterol 2006; 101: $2139-2146$

[2] Lowe AS, Stephenson S, Kay CL et al. Duodenal obstruction by gallstones (Bouveret's syndrome): a review of the literature. Endoscopy 2005; 37: $82-87$

[3] Chaudhary P. Acinar cell carcinoma of the pancreas: a literature review and update. Indian J Surg 2015; 77: 226-231

[4] La Rosa S, Sessa F, Capella C. Acinar cell carcinoma of the pancreas: overview of clinicopathologic features and insights into the molecular pathology. Front Med 2015; 2: 41. DOI: 10.3389/fmed.2015.00041

\section{Bibliography}

DOI http://dx.doi.org/10.1055/s-0042-123499

Endoscopy 2017; 49: E62-E63

(c) Georg Thieme Verlag KG

Stuttgart · New York

ISSN 0013-726X 